\title{
Sulfobetaine methacrylate hydrogel-coated anti-fouling surfaces for implantable biomedical devices
}

\author{
Se Yeong Lee, Yunki Lee, Phuong Le Thi, Dong Hwan Oh and Ki Dong Park
}

\begin{abstract}
Background: Zwitterionic molecules have been widely studied as coating materials for preparing anti-fouling surfaces because they possess strong hydration properties that can resist non-specific protein adsorption. Numerous studies on surface modification using zwitterionic molecules have been investigated, such as electrochemically mediated and photoinitiated radical polymerization. However, these methods have some limitations, including multi-step process, difficulties in producing thick and dense layers as well as the requirement of extra facilities. In this study, we report a novel zwitterionic hydrogel-coating method via Fenton reaction for the preparation of anti-fouling surfaces.

Methods: Sulfobetaine methacrylate (SBMA) hydrogel was coated on polyurethane (PU) by polymerization of SBMA molecules via the Fenton reaction. The coated surfaces were characterized by the measurements of water contact angle, SEM and XPS. The anti-fouling properties of the modified surfaces were evaluated by reductions of fibrinogen absorption and cell (human dermal fibroblasts, hDFBs) adhesion.

Results: SBMA hydrogel layers were coated on the PU substrates and these layers have a high affinity for water. The hydrogel coatings were highly stable for 7 days, without a significant change in surface wettability. Importantly, the hydrogel-coated PU substrates decrease $80 \%$ of surface-adsorbed fibrinogen and surface-attached hDFBs (compared with uncoated PU substrates), indicating the excellent anti-fouling activities of modified surfaces.
\end{abstract}

Conclusions: The hydrogel-coated PU surfaces prepared by Fenton reaction with anti-fouling properties could have potential uses for implantable biomedical devices.

Keywords: Surface modification, Zwitterions, Anti-fouling properties, Fenton reaction, Hydrogel

\section{Background}

Biomaterials are inert natural or synthetic materials that come in contact with tissue, blood or biological fluids, and are intended for use in prosthetic, diagnostic, therapeutic or storage applications without adversely affecting the living organism and its components [1]. There are various types of biomaterials, such as metals, ceramics and polymers. The biomaterials have been widely used for biomedical applications, such as blood-contacting devices, biosensors, drug delivery vehicles and other implantable devices. When being implanted into the body, various reactions can be happened between the host body and biomaterials. For examples, the non-specific protein adsorption onto the

\footnotetext{
* Correspondence: kdp@ajou.ac.kr

Department of Molecular Science and Technology, Ajou University, San 5, Woncheon, Yeongtong, Suwon 16499, Republic of Korea
}

implant surfaces, subsequently aiding the blood cell adhesion, which leads to the thrombus formation and causes detrimental clinical complications [2]. In this context, surface modification with protein/cell-resistant properties is an effective strategy to improve the in vivo performance of biomaterials used for blood-contacting devices [3-5]. Through a proper modification technique, the biomaterial surfaces can be physically or chemically modified to minimize protein adsorption and cell adhesion.

Hydrophilic polymers such as poly(2-hydroxyethyl methacrylate) and poly(ethylene glycol) (PEG) are routinely used to modify surfaces. After being immobilized on the surfaces, these materials create a hydration layer on the surfaces, thereby improving the protein-resistant properties of the modified surfaces. Although the antifouling properties of PEGylated surfaces were greatly 
enhanced, several disadvantages have been reported for in vivo application, such as the relatively poor performance in blood serum/plasma and susceptibility to oxidation $[6,7]$. Zwitterionic polymers, a family of materials possessing strong hydration properties that effectively resist nonspecific protein adsorption, have been extensively studied for preparing anti-fouling surfaces [8-10]. Compared to PEG, zwitterionic polymers are not only biomimetic but also biocompatible and non-cytotoxic, as their endotoxin levels were found to be acceptable for in vivo implantation [11]. Numerous approaches are available to modify a surface using zwitterionic molecules, such as photoinitiated cross-linking and electrochemically mediated radical polymerization. However, these methods have some limitations, including multi-step process, use of toxic reagents, difficulties in producing thick and very dense layers, in addition to the requirement of extra facilities [12-15].

Recently, free radicals generated by redox systems under mild conditions have been applied to initiate the crosslinking process. Among them, the redox pair of ferrous salt and hydrogen peroxide (Fenton reaction) produces highly reactive hydroxyl radicals, as shown in the equation below [16]:

$$
\mathrm{Fe}^{2+}+\mathrm{H}_{2} \mathrm{O}_{2} \rightarrow \mathrm{Fe}^{3+}+\mathrm{OH}^{-}+\mathrm{OH}^{-}
$$

Nowadays, the Fenton reaction is used to handle water pollution [17]. Also, Fenton's reagent has been used to induce radical polymerization of vinylic molecules for more than half century [18]. Fenton reaction was recently explored to prepare poly (N-vinyl-2-pyrrolidone) (PVP) hydrogels, which did not show any toxic or disturbing outcomes based on a dermal inflammation test in rabbits [19].

Herein, we report a simple and effective method to develop anti-fouling surfaces, by coating a zwitterionic hydrogel onto polyurethane (PU) substrates. We hypothesized that the high hydration of both the zwitterionic polymer and hydrogel network would suppress the protein absorption and thus prevent the unwanted reactions between cells and surfaces. The hydrogel was rapidly formed by Fenton reaction-initiated free radical polymerization of sulfobetaine methacrylate (SBMA) and ethylene glycol dimethacrylate (EGDMA). The physicochemical properties of the modified surface such as surface morphology, chemical composition, and surface wettability were characterized by scanning electron microscopy (SEM), X-ray photoelectron spectroscopy (XPS), and water contact angle measurement, respectively. The anti-fouling activities of hydrogel coated surfaces were investigated by in vitro protein adsorption and cell attachment.

\section{Methods}

\section{Materials}

PU (Estane, 60D, Lubrizol Corporation, Wickliffe, USA) was kindly provided by Genoss, Suwon, Korea. SBMA, iron (II) chloride $\left(\mathrm{FeCl}_{2}\right)$, L-ascorbic acid (AA), cumene hydroperoxide (CHP), ethylene glycol dimethacrylate (EGDMA), hexane, sodium dodecyl sulfate (SDS), and tetrahydrofuran (THF) were obtained from Sigma-Aldrich (St. Louis, MO, USA). N, $\mathrm{N}$-dimethylacetamide (DMAc) was supplied by Junsei Chemical Co. (Tokyo, Japan). All other chemicals were purchased from Sigma-Aldrich unless otherwise specified.

For the protein adsorption measurement, horseradish peroxidase (HRP)-conjugated anti-IgG, fibrinogen, antifibrinogen, 3,3',5,5'-tetramethylbenzidine (TMB), bovine serum albumin (BSA) and sulfuric acid were purchased from Sigma-Aldrich. For the cell study, Dulbecco's modified Eagle's medium (DMEM), fetal bovine serum (FBS), penicillin-streptomycin $(\mathrm{P} / \mathrm{S})$, trypsin/ethylenediaminetetraacetic acid (EDTA) and Dulbecco's phosphate buffered saline were purchased from Gibco BRL (Grand Island, NY, USA). The DNA-specific fluorochromes, 4'6-diamidino-2-phenylindole (DAPI) was procured from Vector Laboratory (Burlingame, CA, USA).

\section{Preparation of PU substrates}

PU substrates were prepared using casting and solvent evaporation. PU pellets were dissolved in a co-solvent of THF:DMAc $(50: 50, v / v)$ at $60{ }^{\circ} \mathrm{C}$. The PU solution $(10 \%$ $w / v)$ was poured into a glass mold at $60{ }^{\circ} \mathrm{C}$ and dried under vacuum for $48 \mathrm{~h}$. Before modification, PU substrates were cut out with a metal stamp and washed with deionized water (DW, $48 \mathrm{~h}$ ), to extract residual solvents. The thickness of PU substrates was measured using a digital caliper (Mitutoyo Co., Japan).

\section{Preparation of SBMA hydrogel-coating on PU substrates}

Hydrogel-coated PU substrates were fabricated by a twostep process (Fig. 1). First, the PU substrates were immersed in a hexane solution containing EGDMA (5\%, $v / \mathrm{v})$ and CHP $(20 \%, \mathrm{v} / \mathrm{v})$ for $5 \mathrm{~min}$ at room temperature (RT). Then, the samples were placed into a solution containing SBMA $(50 \%, w / v), \mathrm{FeCl}_{2}(0.1 \%, w / v)$, and $\mathrm{AA}$ (1\%. w/v) for $15 \mathrm{~min}$ at RT. After the coating procedure, the hydrogel-coated PU substrates were washed with $1 \%$ (w/v) SDS solution for 5 min to remove unbound SBMA and immersed in DW overnight.

\section{Surface characterization of SBMA hydrogel coated PU substrates}

The morphology of the hydrogel-coated PU substrates was examined by scanning electron microscopy (SEM, S-800, Hitachi). Briefly, the substrates were affixed onto aluminum stubs using double-sided adhesive conductive carbon tape. Before imaging, the samples were coated with a very thin layer of gold. The images were captured under high vacuum conditions at $5 \mathrm{kV}$.

The hydrophilicity of the modified surface was measured by the sessile drop method, using a contact angle 


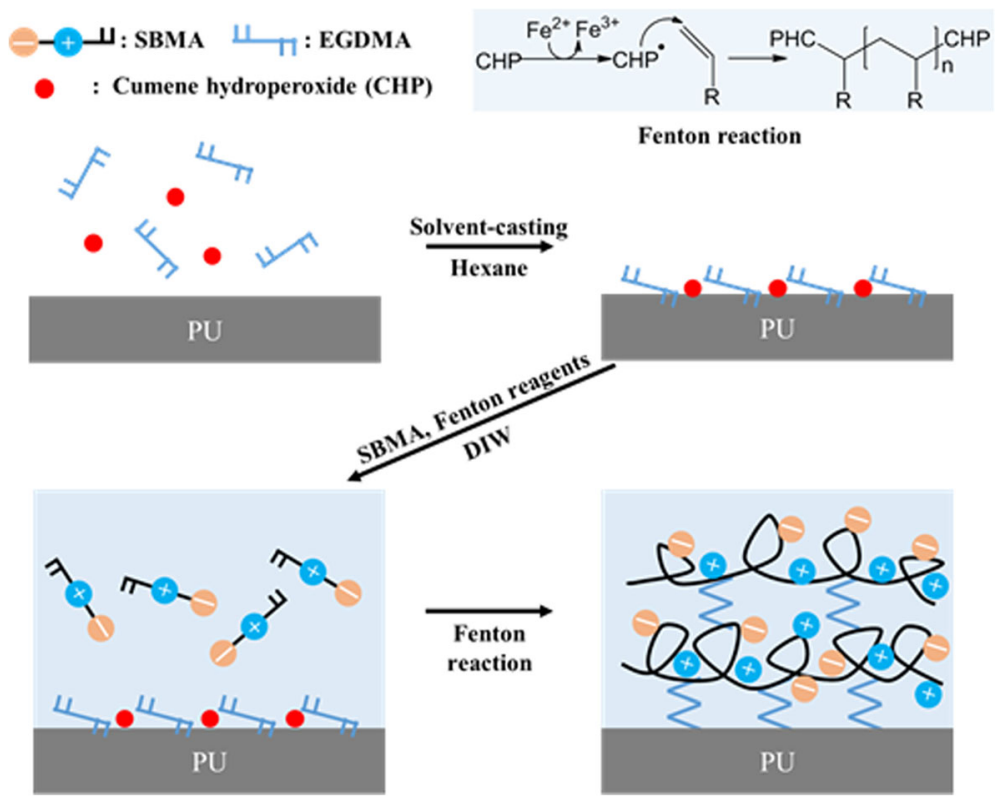

Fig. 1 Schematic presentation for preparation of SBMA hydrogel-coated PU substrates

goniometer (GBX Inc., France). A $1 \mu \mathrm{L}$ water drop was placed on the surface using a micro-syringe. The water contact angles were measured at different positions on the surface after $30 \mathrm{~s}$ of dropping.

The surface composition of the hydrogel-coated PU substrates was analyzed by X-ray photoelectron spectroscopy (XPS) (Thermo Electron, K-Alpha, USA). The $\mathrm{C}_{1 \mathrm{~s}}$ hydrocarbon peak at $284.84 \mathrm{eV}$ was used as the reference for all binding energies. Measured peak areas were converted to normalized peak intensities by atomic sensitivity factors, from which the atomic compositions of surfaces were calculated.

\section{Stability of hydrogel coating}

The stability of the hydrogel coating on PU surfaces was investigated by measurement of the water contact angle using the contact angle goniometer as described above. Before the analysis, the coated surfaces were immersed in distilled water for predetermined time periods $(0,1,3$, 5, 7, 14 and 21 days), and then air-dried overnight at RT.
In vitro anti-fouling evaluation

\section{Fibrinogen adsorption}

The fibrinogen adsorption onto the bare and SBMA hydrogel coated PU surfaces was evaluated using the the antigen-antibody reaction method. Fibrinogen was dissolved in PBS at $1 \mathrm{mg} / \mathrm{mL}$. The surfaces were first immersed in PBS solution for $30 \mathrm{~min}$ to achieve equilibrium and then transferred to a 24-well plate which contained $500 \mu \mathrm{L}$ of fibrinogen solution per well. The experiment was preceded at $37^{\circ} \mathrm{C}$ for $3 \mathrm{~h}$. Next, the samples were washed $(\times 3)$ with PBS solution containing 0.05 wt.\% TWEEN 20 (PBST) for $5 \mathrm{~min}$. In order to prevent the noise signal from non-specific fouling, the samples were blocked with $2 \mathrm{mg} / \mathrm{mL}$ BSA in PBST for $30 \mathrm{~min}$. Afterward, the samples were separately immersed in solutions containing anti-fibrinogen at 1:10,000 dilution for $1 \mathrm{~h}$ at RT. After removal from the primary antibody solutions, the samples were washed $(\times 3)$ with PBST for $5 \mathrm{~min}$, followed by incubation in corresponding HRPconjugated IgG at 1:10,000 dilution for $1 \mathrm{~h}$ at RT. After
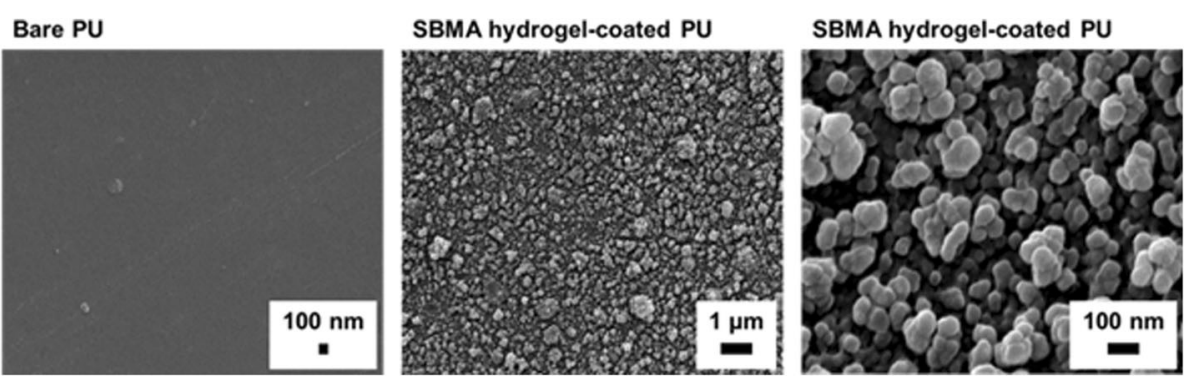

Fig. 2 Surface morphology of PU and SBMA hydrogel-coated PU substrates 

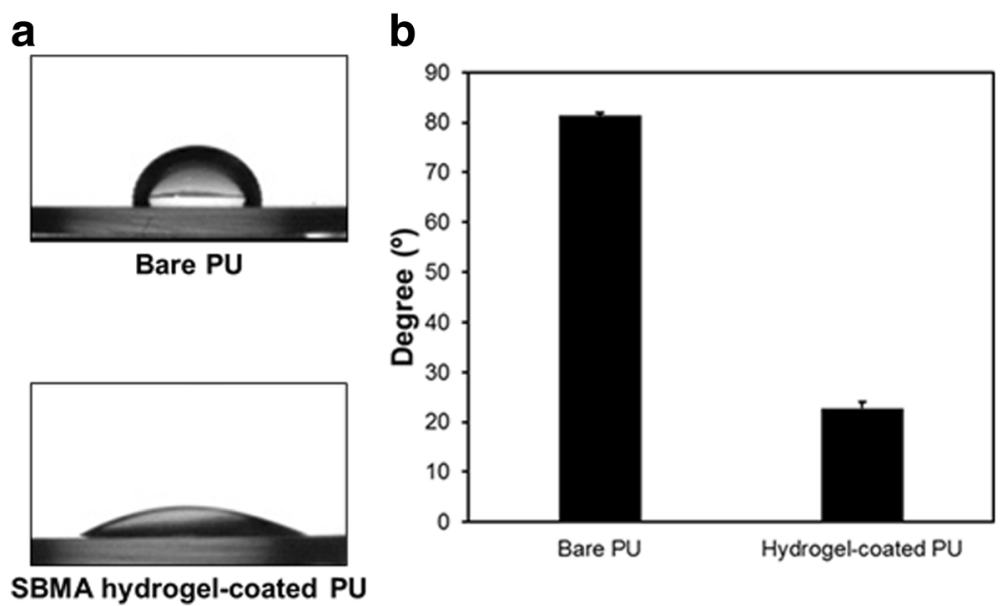

Fig. 3 Surface wettability (a) and water contact angles (b) of bare and SBMA hydrogel-coated PU substrates

washing $(\times 3)$ with PBST for 5 min, the samples were reacted with TMB substrate for $10 \mathrm{~min}$ and the reaction was stopped with $\mathrm{H}_{2} \mathrm{SO}_{4}\left(1 \mathrm{M}, \mathrm{TMB}: \mathrm{H}_{2} \mathrm{SO}_{4}=2: 1 v / \mathrm{v}\right)$. The optical density (OD) of the supernatants was read at $450 \mathrm{~nm}$ by a microplate reader (SpectraMax, Molecular Devices, Sunnyvale, CA) to determine the amount of adsorbed fibrinogen.

\section{Cell adhesion}

To evaluate cell adhesion onto the bare and hydrogelcoated PU substrates, human dermal fibroblasts (hDFBs) were seeded on the PU surfaces at a density of $2 \times 10^{4}$ cells $/ \mathrm{cm}^{2}$. The cell-seeded PU substrates were cultured with DMEM supplemented with 10\% FBS containing 1\% $\mathrm{P} / \mathrm{S}$, under standard cell culture conditions $\left(5 \% \mathrm{CO}_{2}\right.$, at $37^{\circ} \mathrm{C}$ ). After $6 \mathrm{~h}$ of incubation, blue nucleic acid staining was performed with DAPI which preferentially bind to A (adenine) and $\mathrm{T}$ (thymine) regions of DNA. The DAPIstained cells were imaged by fluorescence microscopy (TE-2000, Nikon, Japan).
Automatic cell counting in the fluorescence images was performed using the ImageJ software (NIH, Bethesda, MD, USA). Five images per samples were used to analyze the cell number by counting the nuclei of cells. The entire area of the image was calculated and the result was presented as the number of cells per unit area of a sample.

\section{Statistical analyses}

Experimental data were analyzed by the Student's t-test. Statistical significance was set at having " $P<0.05$. All the experiments were performed in triplicate, and data were presented as the mean $\pm \mathrm{SD}$.

\section{Results and discussion}

Surface characterization of SBMA hydrogel-coated PU substrates

The morphology of bare and hydrogel-coated PU surfaces was visualized by SEM. As shown in Fig. 2, the surface roughness increased significantly after coating
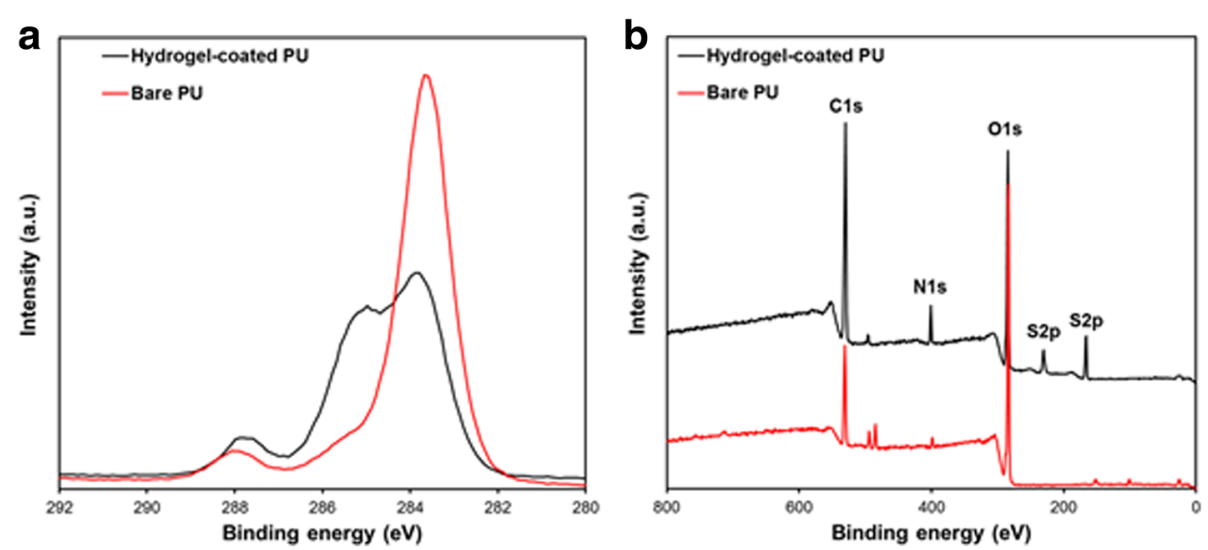

Fig. 4 XPS $C_{15}$ core-level spectra (a) and XPS wide-scan spectra (b) of bare and SBMA hydrogel-coated PU surfaces 
Table 1 Surface chemical composition of bare and SBMA hydrogel-coated PU substrates

\begin{tabular}{llllll}
\hline Sample & \multicolumn{4}{l}{ Atomic percentage (\%) } & \multirow{2}{*}{ Atomic ratio } \\
\cline { 2 - 5 } & C1s & O1s & N1 s & S2p & N/S ratio \\
\hline Bare PU & 85.64 & 13.25 & 1.11 & 0 & - \\
Hydrogel-coated PU & 64.16 & 25.63 & 4.71 & 5.5 & 0.86 \\
\hline
\end{tabular}

SBMA, sulfobetaine methacrylate; PU, polyurethane

hydrogel onto the PU surfaces. Furthermore, the hydrogel coating was composed of small SBMA spheres, which adhered to each other. This result was explained by the effect of EGDMA cross-linkers, which could interact with two SBMA chains and lead to the formation of a sphere-like network [20].

The hydrophilicity of the PU surfaces was investigated before and after coating with the SBMA hydrogel, using the static water contact angle measurement. Figure 3 shows the dramatic decrease in the water contact angle of the SBMA hydrogel-coated PU surfaces $\left(81.5^{\circ} \pm 0.6^{\circ}\right)$ compared to the bare PU ones $\left(22.7^{\circ} \pm 1.4^{\circ}\right)$. This result indicated the successful coating of hydrophilic zwitterionic hydrogel onto the PU substrates.

The difference in chemical composition between the bare and hydrogel-coated surfaces was determined by XPS analysis. Figure 4a shows the XPS $\mathrm{C}_{1 \mathrm{~s}}$ core-level spectra processed via curve fitting. There was a high binding energy peak at $288 \mathrm{eV}$, corresponding to the $\mathrm{O}$ $\mathrm{C}=\mathrm{O}$ species. The main $\mathrm{C}_{1 \mathrm{~s}}$ peak for SBMA is attributed to the overlap of $\mathrm{CO}, \mathrm{CN}^{+}, \mathrm{CC}$, and $\mathrm{CSO}_{3}^{-}(\sim 285.2 \mathrm{eV})$ [21]. In the wide-scan XPS spectra (Fig. 4b), after the polymerization of SBMA onto the surface of PU substrates, the new peaks appeared at around $401.1 \mathrm{eV}$ $\left(\mathrm{N}_{1 \mathrm{~s}}\right)$ and $167.1 \mathrm{eV}\left(\mathrm{S}_{2 \mathrm{p}}\right)$, which originated from the $-\left(\mathrm{CH}_{3}\right)_{3} \mathrm{~N}^{+}$and $-\mathrm{SO}_{3}^{-}$groups of SBMA molecules. These

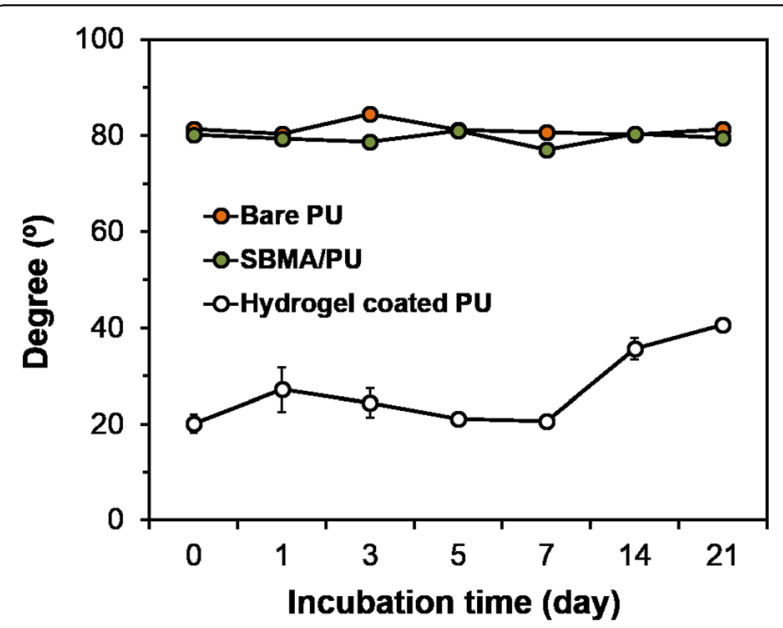

Fig. 5 Water contact angle of bare and modified PU surfaces, after incubating in aqueous solution data demonstrated that SBMA hydrogel was successfully coated onto the PU surface. Both the nitrogen and sulfur compositions calculated from XPS spectra concur with the stoichiometric compositions (Table 1).

\section{Stability of hydrogel coating}

The stability of the hydrogel coatings was analyzed by measuring the hydrophilicity of modified PU substrates during a 21-day incubation in aqueous solution. It was observed that the SBMA hydrogel-coated PU substrates had a high stability in aqueous condition (Fig. 5), with no marked change in the water contact angle (around $20^{\circ}$ ) after incubation for 7 days. At this time, the water contact angle of the hydrogel-coated PU surfaces slightly increased up to $40^{\circ}$, but was still lower than that of bare PU surfaces $\left(81^{\circ}\right)$ and PU substrates treated with only SBMA solution (SBMA/PU). From the above results, we can confirm the high stability of the Fenton reactionmediated coating method.

\section{In vitro anti-fouling evaluation Fibrinogen adsorption}

The protein adsorption of SBMA hydrogel-coated PU substrates was evaluated using HRP-conjugated anti-IgG (by an indirect ELISA). The amount of anti-IgG adsorption on SBMA hydrogel-coated PU substrates was determined by monitoring the increase in tangerine color intensity at $450 \mathrm{~nm}$, caused by the reaction of HRP with TMB, relative to bare PU. The sensitivity of the ELISA method was found to be equivalent to that of the ${ }^{125}$ I-radiolabeled fibrinogen method for estimating an adsorption level [22].

In this study, the absorbance from the bare $\mathrm{PU}$ substrate was set as $100 \%$ for calculating the relative adsorption. As shown in Fig. 6, there was a significant decrease in fibrinogen absorption on SBMA hydrogelcoated PU surfaces, compared to the bare PU surfaces. This result confirmed the effect of the zwitterion-based hydrogel with its well-packed structure, to reduce the non-specific protein adsorption on the PU surface. It is well-known that the adsorption of protein on the implant surfaces is the first step in thrombus formation, one of the common and severe events in bloodcontacting devices [2]. Therefore, the reduction in fibrinogen absorption, the key protein of the coagulation cascade, making our SBMA hydrogel-coated surface a potential candidate for in vivo biomedical applications.

\section{Cell adhesion}

The cell attachment response on the material surface plays an important role in designing various types of biomedical devices and usually needs to be prevented by proper surface modification. The cell-repellent properties of the SBMA hydrogel-coated PU surfaces were 

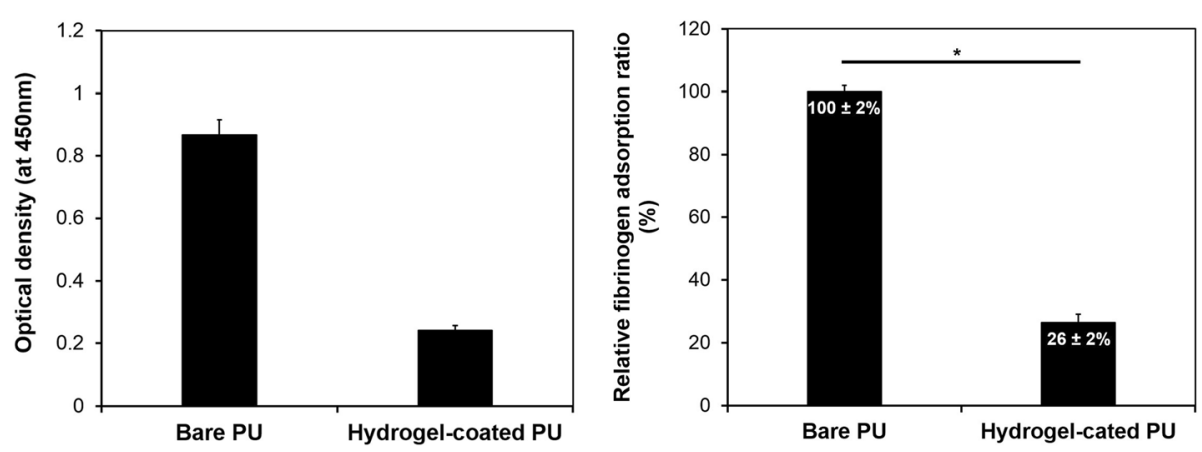

Fig. 6 Fibrinogen absorption on bare and hydrogel-coated PU surfaces: optical density of absorbed fibrinogen solution (a) and relative fibrinogen absorption ratio, compared to bare surfaces $(\mathbf{b}) .{ }^{*} P<0.0001$ vs. bare PU

tested using hDFBs as a model cell line and bare PU as the control. Figure $7 \mathrm{a}$ shows the representative fluorescence images of the hDFBs adhered on the bare and SBMA hydrogel-coated PU surfaces. While the cells adhered well to the bare PU surfaces, the SBMA hydrogel-coated PU surfaces showed relatively less adherence. The quantitative statistics of the attached hDFBs was also obtained from ImageJ analysis (Fig. 7b). Compared to the bare PU, the SBMA hydrogel-coated PU surfaces suppressed $80 \%$ of adhered hDFBs (Fig. 7c). The reduction in cell adhesion of the hydrogel-coated surfaces may result from the cytotoxicity of the hydrogel coating. However, the excellent biocompatibility of SBMA hydrogels and Fenton cross-linking reaction has been widely demonstrated $[6,19,23,24]$. In our study, the modified surfaces were washed thoroughly with SDS and immersed in DW overnight, to remove the solvent and unreacted monomers. In addition, the cell-repellent property of our hydrogel-coated surfaces corroborated with and was comparable to previous studies, which reduced over $75 \%$ cell attachment relative to bare surfaces $[9,25]$. These results are most likely due to the

\section{a}

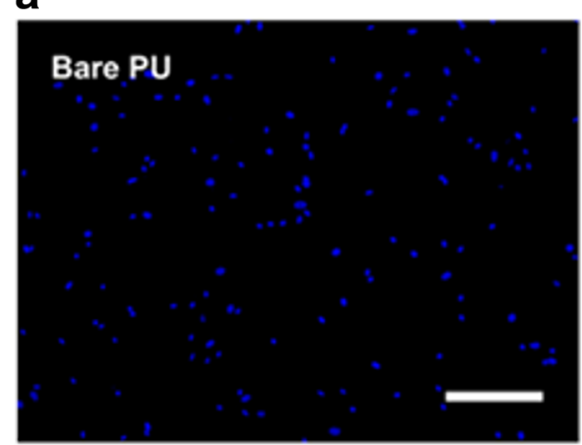

\section{SBMA hydrogel-coated PU}
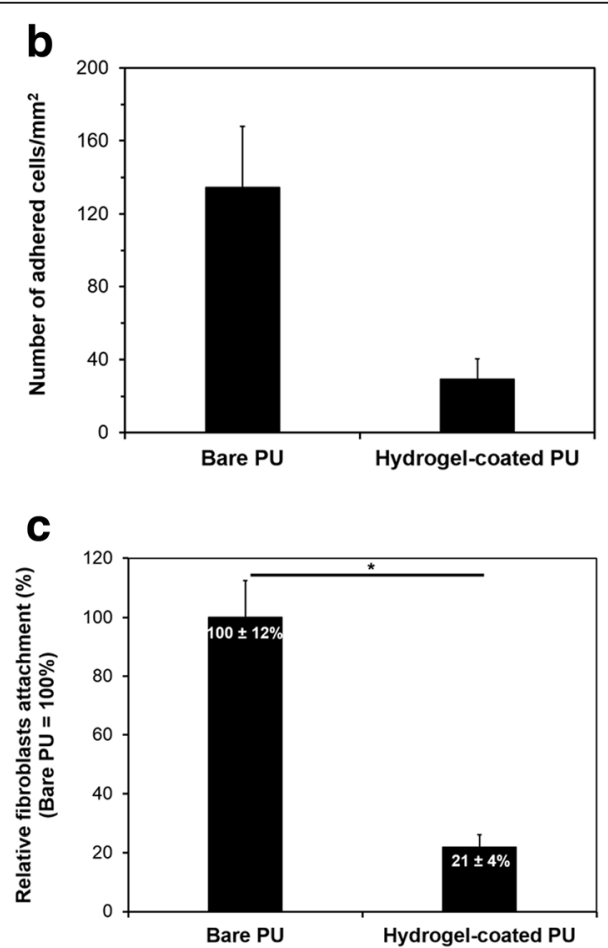

Fig. 7 In vitro fibroblast adhesion on bare and hydrogel coated PU surfaces: Fluorescence micrographs of cells adhered to the bare and SBMA hydrogel-coated PU surfaces (a); amount of attached cells measured by ImageJ analysis (b) and relative cell attachment ratio, compared to bare surfaces $(\mathbf{c}) .{ }^{*} P<0.01$ vs. bare PU 
hydration properties and the lack of electrostatic properties of the SBMA hydrogel coating, deeming it resistant to protein absorption before cell attachment.

\section{Conclusions}

In this study, we developed a facile method to polymerize and coat a zwitterionic-based hydrogel onto PU surfaces via the Fenton reaction. By using the two-phase process, the free radicals were formed on the PU surface/reaction solution interface, which improves the efficiency of polymerization. The obtained hydrogel coating showed good adhesion to the PU surface with high stability after 21 days of incubation in aqueous solution. Importantly, the SBMA hydrogel-coated PU surfaces exhibited good resistance against both fibrinogen and fibroblasts, attributed to the excellent anti-fouling properties of zwitterionic molecules. Therefore, the SBMA hydrogel-coated PU surfaces with anti-fouling properties could have great potential for use in biomedical devices.

\section{Acknowledgements}

Not applicable.

\section{Funding}

This research was supported by the Bio \& Medical Technology Development Program of the NRF funded by the Korean government, MSIP (NRF2015M3A9E2028578); and the Materials and Components Technology Development Program (Strategic Core Material Technology Development Program) of MOTIE/KEIT. [10053595, Development of functionalized hydrogel scaffold based on medical grade biomaterials with $30 \%$ or less of molecular weight reduction].

\section{Availability of data and materials}

All data generated or analyzed during this study are included in this published article.

\section{Authors' contributions}

The research was designed and coordinated by KDP. The experiment and analysis were performed by SYL. The manuscript was improved and revised by YKL and PLT. All authors have read and approved the final manuscript.

Ethics approval and consent to participate

Not applicable.

\section{Consent for publication}

All authors consent for publication.

\section{Competing interests}

The authors declare that they have no competing interests.

\section{Publisher's Note}

Springer Nature remains neutral with regard to jurisdictional claims in published maps and institutional affiliations.

Received: 23 November 2017 Accepted: 20 December 2017 Published online: 12 February 2018

\section{References}

1. Langton CM, Njeh CF, Hodgskinson R, Currey JD. Prediction of mechanical properties of the human calcaneus by broadband ultrasonic attenuation. Bone. 1996;18:495-503.
2. Adipurnama I, Yang MC, Ciach T, Butruk-Raszeja B. Surface modification and endothelialization of polyurethane for vascular tissue engineering applications: a review. Biomater Sci. 2016;5:22-37.

3. Chen C, Bang S, Cho Y, Lee S, Lee I, Zhang S, Noh I. Research trends in biomimetic medical materials for tissue engineering: 3D bioprinting, surface modification, nano/micro-technology and clinical aspects in tissue engineering of cartilage and bone. Biomater Res. 2016;20:10.

4. Damodaran VB, Murthy NS. Bio-inspired strategies for designing antifouling biomaterials. Biomater Res. 2016;20:18.

5. Han JW, Shin YS, Kim JJ, Son HS. Comparison of in vivo antibacterial and antithrombotic activities of two types of pulmonary artery catheters in pig Biomater Res. 2017;21:23

6. Ye L, Zhang Y, Wang Q, Zhou X, Yang B, Ji F, Dong D, Gao L, Cui Y, Yao F. Physical cross-linking starch-based zwitterionic hydrogel exhibiting excellent biocompatibility, protein resistance, and biodegradability. ACS Appl Mater Interfaces. 2016;8:15710-23.

7. Kwon HJ, Lee Y, Phuong LT, Seon GM, Kim E, Park JC, Yoon H, Park KD. Zwitterionic sulfobetaine polymer-immobilized surface by simple tyrosinase-mediated grafting for enhanced antifouling property. Acta Biomater. 2017;61:169-79.

8. Hu Y, Yang G, Liang B, Fang L, Ma G, Zhu Q, Chen S, Ye X. The fabrication of superlow protein absorption zwitterionic coating by electrochemically mediated atom transfer radical polymerization and its application. Acta Biomater. 2015;13:142-9.

9. BY Y, Zheng J, Chang Y, Sin MC, Chang $C H$, Higuchi A, Sun YM. Surface zwitterionization of titanium for a general bio-inert control of plasma proteins, blood cells, tissue cells, and bacteria. Langmuir. 2014:30:7502-12.

10. Quintana R, Janczewski D, Vasantha VA, Jana S, Lee SS, Parra-Velandia FJ, Guo S, Parthiban A, Teo SL, Vancso GJ. Sulfobetaine-based polymer brushes in marine environment: is there an effect of the polymerizable group on the antifouling performance? Colloids Surf B. 2014;120:118-24.

11. Zhang Z, Chao T, Liu L, Cheng G, Ratner BD, Jiang S. Zwitterionic hydrogels: an in vivo implantation study. J Biomater Sci Polym Ed. 2009;20:1845-59.

12. Liu F, Hashim NA, Liu Y, Abed MRM, Li K. Progress in the production and modification of PVDF membranes. J Memb Sci. 2011;375:1-27.

13. Zhao $Y H$, Zhu XY, Wee KH, Bai R. Achieving highly effective non-biofouling performance for polypropylene membranes modified by UV-induced surface graft polymerization of two oppositely charged monomers. J Phys Chem B. 2010;114:2422-9.

14. Hu S, Brittain WJ. Surface grafting on polymer surface using physisorbed free radical initiators. Macromolecules. 2005;38:6592-7.

15. Joung YK, Choi JH, Bae JW, Park KD. Hyper-branched poly(poly(ethylene glycol)methacrylate)-grafted surfaces by photo-polymerization with iniferter for bioactive interfaces. Acta Biomater. 2008:4:960-6.

16. Sarac AS. Redox polymerization. Prog Polym Sci. 1999;24:1149-204.

17. Tokumura M, Wada Y, Usami Y, Yamaki T, Mizukoshi A, Noguchi M, Yanagisawa Y. Method of removal of volatile organic compounds by using wet scrubber coupled with photo-Fenton reaction - preventing emission of by-products. Chemosphere. 2012;89:1238-42.

18. Gambogi RJ, Cho DL, Yasuda H, Blum FD. Characterization of plasma polymerized hydrocarbons using CP-MAS 13C-NMR. J Polym Sci A. 1991;29:1801-5.

19. Barros JAG, Fechine GJM, Alcantara MR, Catalani LH. Poly(N-vinyl-2-pyrrolidone) hydrogels produced by Fenton reaction. Polymer. 2006;47:8414-9.

20. Rong Q, Han H, Feng F, Ma Z. Network nanostructured polypyrrole hydrogel/au composites as enhanced electrochemical biosensing platform. Sci Rep. 2015;5:11440.

21. Zhang Z, Chao T, Chen S, Jiang S. Superlow fouling sulfobetaine and carboxybetaine polymers on glass slides. Langmuir. 2006;22:10072-7.

22. Bergstrom K, Holmberg K, Safranj A, Hoffman AS, Edgell MJ, Kozlowski A, Hovanes BA, Harris JM. Reduction of fibrinogen adsorption on PEG-coated polystyrene surfaces. J Biomed Mater Res. 1992;26:779-90.

23. Sun L, Zhang S, Zhang J, Wang N, Liu W, Wang W. Fenton reaction-initiated formation of biocompatible injectable hydrogels for cell encapsulation. Mater Chem B. 2013;1:3932.

24. Kao C-W, Cheng P-H, P-T W, Wang S-W, Chen IC, Cheng N-C, Yang K-C, Zwitterionic YJ. Poly(sulfobetaine methacrylate) hydrogels incorporated with angiogenic peptides promote differentiation of human adipose-derived stem cells. RSC Adv. 2017;7:51343-51.

25. Chien HW, Tsai CC, Tsai WB, Wang MJ, Kuo WH, Wei TC, Huang ST. Surface conjugation of zwitterionic polymers to inhibit cell adhesion and protein adsorption. Colloids Surf B. 2013:107:152-9. 